\title{
Probabilistic Modeling of Delays in Data Transmission Systems with Wireless Network Interfaces Employing Random Strings Function
}

\begin{abstract}
T. TOPÓR-KAMIŃSKI* AND M. GRYGIEL
Institute of Measurement Science, Electronics and Control, Silesian University of Technology, Gliwice, Poland

The paper presents the concepts of model construction on transmission delays in systems with wireless interfaces using random series of function. It is a new method of modelling that allows us to use probabilistic description of time required to realise tasks in various devices. It also allows us to use time domain delta function series in order to describe the time of transmission of data between transmitter and receiver. In description of delays probability density function is applied. The model also takes into account existence of intermediary network devices that play a significant role in overall budget of delay.
\end{abstract}

DOI: $10.12693 /$ APhysPolA.124.578

PACS: 02.50.-r, 06.90.+v

\section{Introduction}

Common use of wireless networks in controlmeasurement data transmission is a reason why users of such networks must take into account many problems such as determining quality of service in these networks. In modern automation systems knowledge of these parameters allows one to design the system properly [1]. One of the parameters determining the quality of data transmission network are delays [2].

Due to its nature particularly prone to delays are wireless networks $[3,4]$. In the wireless network, there are many factors causing delay on the data path from transmitter to receiver. Those delays can be caused by software or hardware factors, and also can be caused by various disturbance factors. In the paper it is assumed that software delays are a sum of two partial delays - own one and additional one associated with concurrent execution of multiple tasks. Moreover when one includes additional hardware delays occurring in the device, then one can say that total delay is a sum of many partial delays [5-8]. Delays can be modelled as a deterministic or a probabilistic [9].

Probabilistic analysis of network delay in data control and measurement allows us to determine distribution of delays resulting from random described factors $[5,6]$. They have a direct impact on the operation of the entire automation system using a data network to control the individual components [1].

This kind of description is a natural consequence of the fact that the decisions to establish communications in the network are random. In addition, the probabilistic description of delay provides full information about its properties, and thus it can be used to analyse the

\footnotetext{
* corresponding author; e-mail: tadeusz. topor-kaminski@polsl.pl
}

properties of the network in a much broader way than the deterministic description [6].

\section{Mathematical basis of the proposed probabilistic modeling of delays}

Described below model of communication delays used in wireless networks is based on mathematical apparatus presented in [5]. This model allows the description of delay when the transfer takes place from the point of origin (transmitter) to point of destination (receiver) including any intermediary network devices in transmission.

Operation of algorithm CSMA/CA and the fact that the starting point of transmission is not clearly established, secures a probabilistic description of delays [3-5]. This model also describes the phenomena of repetition rate arising due to communication errors - retransmissions.

Let us consider a simple case. Two devices communicate directly using a wireless network. One of them is a transmitter $\mathrm{A}$ and a second one is a receiver $\mathrm{B}$. Between them there are no other devices or obstacles. Let us assume that $t_{\mathrm{A}}$ represents the time used by node $\mathrm{A}$ to form data in order to send them with wireless interface, $t_{\text {START }}$ represents a time when node A obtained the availability of access to the medium and is clear to send data.

In such case, the delay in the transmission of data related to the time required for the execution of tasks by the transmitting device $\mathrm{A}$ can be written as

$$
\tau_{\mathrm{A}}=t_{\mathrm{A}}-t_{\mathrm{START}} .
$$

In addition, let us assume that the $\tau_{\mathrm{AB}}$ represents the delay caused by sending the data with wireless medium. Total delay caused by such network structure, can be written as follows:

$$
\tau_{\text {tot }}=\tau_{\mathrm{A}}+\tau_{\mathrm{AB}} .
$$

In more complex situation when wireless network operates in star, tree, or mesh configuration total delay is affected by the presence of intermediary network devices. Such situation is shown in Fig. 1. 


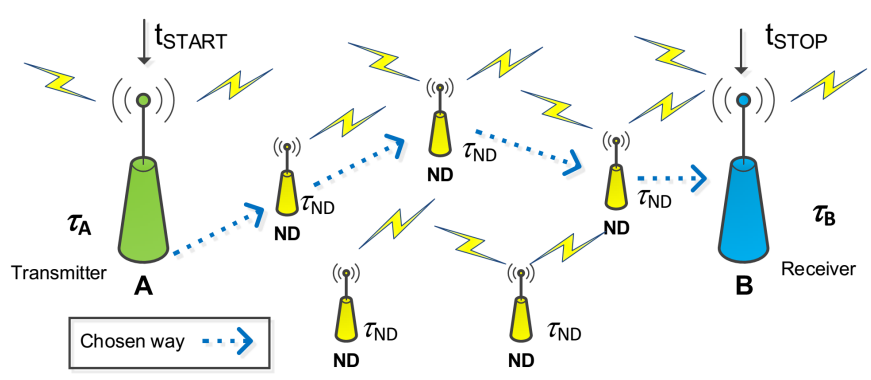

Fig. 1. Sources of delays in data transmission network with wireless interfaces.

Based on notations given in Fig. 1 total delay resulting in data transmission through wireless network folowing the route marked by arrows describes the relationship

$$
\tau_{\text {tot }}=\tau_{\mathrm{A}}+\tau_{\mathrm{AND}}+\tau_{\mathrm{ND}}+\tau_{\mathrm{NDND}}+\tau_{\mathrm{ND}}+\tau_{\mathrm{NDB}},
$$

where $\tau_{\mathrm{ND}}$ represents the delay caused by intermediary network device.

\section{Application of random function keys for description of communication delays}

For the probabilistic description of partial components, one can use the delay probability density function. Probability density function of the delay $\tau_{\mathrm{A}}$ describes the delay related to realisation of software and hardware functions in the transmitter as follows:

$$
g_{\tau_{\mathrm{A}}}=\left\{\begin{array}{cl}
g_{\mathrm{A}}\left(\tau_{\mathrm{A}}\right) & \text { for } \tau_{\mathrm{A} 1} \leq \tau_{\mathrm{A}} \leq \tau_{\mathrm{A} 2}, \text { where } \tau_{\mathrm{A} 1}>0 \\
0 & \text { for other, }
\end{array}\right.
$$

also the probability density function $g_{\tau_{\mathrm{A}}}\left(\tau_{\mathrm{A}}\right)$ is continuous and it satisfies the normalizing condition

$$
\int_{-\infty}^{+\infty} g_{\mathrm{A}}\left(\tau_{\mathrm{A}}\right) \mathrm{d} \tau_{\mathrm{A}}=\int_{\tau_{\mathrm{A} 2}}^{\tau_{\mathrm{A} 1}} g_{\mathrm{A}}\left(\tau_{\mathrm{A}}\right) \mathrm{d} \tau_{\mathrm{A}}=1 .
$$

It is assumed that the probability density function $g_{\tau_{\mathrm{AB}}}\left(\tau_{\mathrm{AB}}\right)$ that describes the distribution of delays caused by the transmission through medium from node $\mathrm{A}$ to node $\mathrm{B}$, is represented by the form

$$
\begin{aligned}
& g_{\tau_{\mathrm{AB}}}\left(\tau_{\mathrm{AB}}\right)=a_{0} \delta\left(\tau_{\mathrm{A}}+\tau_{\mathrm{AB}}-\tau_{0}\right) \\
& \quad+a_{1} \delta\left(\tau_{\mathrm{A}}+\tau_{\mathrm{AB}}-\tau_{1}\right)+a_{k} \delta\left(\tau_{\mathrm{A}}+\tau_{\mathrm{AB}}-\tau_{k}\right),
\end{aligned}
$$

where $\delta$ denotes the Dirac function, defined by:

$$
\delta\left(\tau_{\mathrm{AB}}-\tau_{i}\right)=\left\{\begin{array}{c}
\infty \text { for } \tau_{\mathrm{AB}}=\tau_{i}, i=0,1, \ldots, k, \\
0 \text { for } \tau_{\mathrm{AB}} \neq \tau_{i} .
\end{array}\right.
$$

Moreover, taking into account properties of the Dirac function in relation to $[8,10]$ :

$$
\int_{-\infty}^{+\infty} \delta\left(\tau_{\mathrm{COM}}-\tau_{i}\right) \mathrm{d} \tau_{\mathrm{COM}}=1
$$

where $i=0,1, \ldots, k$ denotes the moment of time. The amount of elements in series is limited to $k$, and the coefficients $a_{0}, a_{1}, \ldots, a_{k}$ are non-negative constants.

Equation (7) is the result of the analysis of the phenomena of multiple access mechanism for the transmission medium, which is implemented on the assumption that in the case of failure to obtain access to the medium a further attempt is made. The maximum number of re- tries is $k$. Specific elements of (7) represent the following attempts of transmission, wherein $\tau_{i}$ indicates the moment of the $i$-th repetition, $i=0,1, \ldots, k$, the coefficient $a_{i}$ determines the probability of transmission repetition $i$.

When the partial delays are independent, the distribution of the total delay, according to the formula (4) can be determined by using the convolution of probability density function for these delays.

In such case, the probability density function of total delay in the transmission of data from the node $\mathrm{A}$ to node $\mathrm{B}$ describes the relationship

$$
g_{\text {tot }}\left(\tau_{\text {tot }}\right)=g_{\mathrm{A}}\left(\tau_{\mathrm{A}}\right) \otimes g_{\mathrm{AB}}\left(\tau_{\mathrm{AB}}\right) .
$$

Convolution is a linear transformation, hence by the introduction of the expression (6) into expression (9) one obtains:

$$
\begin{aligned}
& g_{\text {tot }}\left(\tau_{\text {tot }}\right)=a_{0} g_{\mathrm{A}}\left(\tau_{\mathrm{A}}\right) \otimes \delta\left(\tau_{\mathrm{A}}+\tau_{\mathrm{AB}}-\tau_{0}\right) \\
& +a_{1} g_{\mathrm{A}}\left(\tau_{\mathrm{A}}\right) \otimes \delta\left(\tau_{\mathrm{A}}+\tau_{\mathrm{AB}}-\tau_{1}\right)+\ldots \\
& +a_{k} g_{\mathrm{A}}\left(\tau_{\mathrm{A}}\right) \otimes \delta\left(\tau_{\mathrm{A}}+\tau_{\mathrm{AB}}-\tau_{k}\right) .
\end{aligned}
$$

This expression is the sum of components, each of which is the product of the coefficient $a_{i}$ and the convolution of the delta function with $g_{\mathrm{A}}\left(\tau_{\mathrm{A}}\right)$.

Taking into account the expression (9), one can say that for the $i$-th component expression becomes

$$
g_{\text {tot } i}\left(\tau_{\text {tot }}\right)=g_{\mathrm{A}}\left(\tau_{\mathrm{A}}\right) \otimes \delta\left(\tau_{\text {tot }}-\tau_{i}\right)=g_{\mathrm{A}}\left(\tau_{\text {tot }}-\tau_{i}\right) .
$$

In the expression (11) the product of the convolution of functions corresponds to their Fourier transforms. The Fourier transform of the delta function is equal to 1, while for the function shifted by $\tau$ it is $\mathrm{e}^{-\mathrm{j} \omega t}$. After applying the inverse Fourier transform for Eq. (10) one can get the expression

$$
\begin{aligned}
& g_{\text {tot }}\left(\tau_{\text {tot }}\right)=a_{0} g_{\mathrm{A}}\left(\tau_{\text {tot }}-\tau_{0}\right)+a_{1} g_{\mathrm{A}}\left(\tau_{\text {tot }}-\tau_{1}\right)+\ldots \\
& \quad+a_{k} g_{\mathrm{A}}\left(\tau_{\text {tot }}-\tau_{k}\right) .
\end{aligned}
$$

This means that the probability density function of the total delay is the sum of the products, each of which is composed of two factors. One of them is the probability density function of the delay produced in the node $\mathrm{A}$ shifted by the value $\tau_{i}, i=0,1, \ldots, k$, and the other one is a weighting factor $a_{i}$ describing the weight of the product in the total distribution.

In each of the parts of the expression (12) there is the same probability density function $g_{a}(\cdot)$, wherein in successive parts has different scale factors $a_{i}$ and offset $\tau$. One can, therefore, say that a function $g_{a}(\cdot)$ is a kind of template mapped at different scales for a further parts of expression (12).

In a situation where we have to deal with a situation when the network configuration is more complex, the total delay is the sum of all the delays involved. Following delays occurring in the data path were isolated: $\tau_{\mathrm{A}}$ delay related with an execution of the tasks associated with software and hardware mechanisms as well as with gaining access to the media in the transmitter $\mathrm{A}, \tau_{\mathrm{AND}}-$ delay associated with sending a message from the transmitter A to intermediary network device ND, $\tau_{\mathrm{ND}}-$ delay related with an execution of the tasks associated with software and hardware mechanisms as well as with gain- 
ing access to the media in the network device ND, $\tau_{\mathrm{NDB}}$ - delay associated with sending a message from the intermediary network device ND to the receiver $\mathrm{B}$.

Therefore, the total delay can be described as

$\tau_{\mathrm{COM}}=\tau_{\mathrm{A}}+\tau_{\mathrm{AND}}+\tau_{\mathrm{ND}}+\tau_{\mathrm{NDB}}$.

Probability density function of the transmitter can be described by (6), while the probability density function of intermediary network device can be written as

$$
g_{\tau_{\mathrm{ND}}}\left(\tau_{\mathrm{ND}}\right)=\left\{\begin{array}{l}
g_{\mathrm{ND}}\left(\tau_{\mathrm{ND}}\right) \text { for } \tau_{\mathrm{ND} 1} \leq \tau_{\mathrm{ND}} \leq \tau_{\mathrm{ND} 2}, \\
\quad \text { where } \tau_{\mathrm{ND} 1}>0 \\
0 \quad \text { for other. }
\end{array}\right.
$$

Let us assume that the probability density function describing the distribution of delay caused by the transmission of data from transmitter A to intermediary network device ND is given with expression

$$
\begin{aligned}
& g_{\tau_{\mathrm{AND}}\left(\tau_{\mathrm{AND}}\right)}=a_{0} \delta\left(\tau_{\mathrm{AND}}+\tau_{\mathrm{A}}-\tau_{0}\right) \\
& \quad+a_{1} \delta\left(\tau_{\mathrm{AND}}+\tau_{\mathrm{A}}-\tau_{1}\right)+\ldots \\
& +a_{k} \delta\left(\tau_{\mathrm{AND}}+\tau_{\mathrm{A}}-\tau_{k}\right),
\end{aligned}
$$

where $\delta$ is the symbol of the Dirac function, while $i=$ $0,1, \ldots, k$ a number of times. The number of elements is reduced to a value of $k$, and the weighting factors $a_{0}, a_{1}, \ldots, a_{k}$ are non-negative constants.

Similar formula can be applied for a probability density function describing the delay caused by the transmission of data from intermediary network device ND to a receiver B:

$$
\begin{aligned}
& g_{\tau_{\mathrm{NDB}}}\left(\tau_{\mathrm{NDB}}\right)=b_{0} \delta\left(\tau_{\mathrm{BND}}+\tau_{\mathrm{ND}}-\tau_{0}\right) \\
& \quad+b_{1} \delta\left(\tau_{\mathrm{NDB}}+\tau_{\mathrm{ND}}-\tau_{1}+\ldots\right. \\
& \quad+b_{k} \delta\left(\tau_{\mathrm{NDB}}+\tau_{\mathrm{ND}}-\tau_{k}\right) .
\end{aligned}
$$

Due to a fact that the shift is dependent only on the manufacturer of the devices and for the transmission from the transmitter A to the intermediary network device ND is equal to the time shifting occurring during transmission from intermediary network device ND to the receiver $\mathrm{B}$, the designation for both time shifts is marked as one. According to the mechanism outlined earlier, one can describe total delay as an expression

$$
\begin{aligned}
& g_{\text {tot }}\left(\tau_{\text {tot }}\right)=g_{\mathrm{A}}\left(\tau_{\mathrm{A}}\right) \otimes g_{\mathrm{AND}}\left(\tau_{\mathrm{AND}}\right) \otimes g_{\mathrm{ND}}\left(\tau_{\mathrm{ND}}\right) \\
& \otimes g_{\mathrm{NDB}}\left(\tau_{\mathrm{NDB}}\right) .
\end{aligned}
$$

Substituting Eqs. (4), (14), (15), (16) to (17) and performing the appropriate actions one can show that the total delay is given by the sum of appropriate convolutions.

\section{Identification of partial elements of the total delay}

The delay arising as a result of the implementation of program mechanisms performed during the encapsulation of data to send through the layers of the protocol stack is determined as

$$
\tau_{\mathrm{S}}=t_{\mathrm{end}},
$$

while the delay produced by the action of hardware mechanisms, is as follows:

$$
\tau_{\mathrm{H}}=t_{\text {mod_cod }}+t_{\text {mac }} \text {. }
$$

By using for the description by the probability density functions, one can say that

$$
g_{\tau_{\mathrm{A}}}=g_{\tau_{\mathrm{S}}}\left(\tau_{\mathrm{S}}\right) \otimes g_{\tau_{\mathrm{H}}}\left(\tau_{\mathrm{H}}\right) \text {. }
$$

Then the distribution of delays caused by the operation of the transmitter in order to prepare the data, and the mechanism for access to the medium is a convolution of delays caused by these two mechanisms.

Expression (21) allows us to apply a deconvolution operation in order to determine the parameters that cannot be determined by looking at the construction of the stack or by analysing mechanisms implemented in the standard.

Deconvolution is the inverse operation to the convolution. If the convolution equation is written as [11]:

$$
y(t)=h(t) \otimes x(t)=x(t) \otimes h(t),
$$

then deconvolution allows us to determine the value $x(t)$ for the known values of $y(t)$ and $h(t)$. Introducing estimator

$$
\min _{x} J(t)=\frac{1}{2} E\left\{e^{2}(t)\right\},
$$

where $e(t)=y(t)-y^{\prime}(t)$ and

$$
y^{\prime}(t)=x(t) \otimes h(t)=\sum_{i=0}^{N_{x}} x(i) h(t-i),
$$

where $x(t)$ acts as a filter and $h(t)$ is a known input signal

Using the orthogonally condition (when $h \rightarrow x$ ) [7]:

$$
\begin{aligned}
& \frac{\partial}{\partial x(j)} J(t)=E\left\{e(t) \frac{\partial e(t)}{\partial x(j)}\right\}=0 \\
& \quad \text { for } j=0,1, \ldots, N_{x},
\end{aligned}
$$

then the gradient of the error is equal to

$$
\begin{aligned}
& \frac{\partial e(t)}{\partial x(j)}=\frac{\partial}{\partial x(j)}\left(y(t)-y^{\prime}(t)\right) \\
& \quad=\frac{\partial}{\partial x(j)}\left[y(t)-\sum_{i=0}^{N_{x}} x(i) h(t-i)\right] \\
& \quad=h(t-j) .
\end{aligned}
$$

Substituting (24) to (25) leads to the following formula:

$$
\begin{aligned}
& E\{y(t) h(t-j)\}-\sum_{i=0}^{N_{x}} x(i) E\{h(t-i) h(t-j)\}=0 \\
& \quad \text { for } j=0,1, \ldots, N_{x} .
\end{aligned}
$$

By solving one gets

$$
\sum_{i=0}^{N_{x}} x(i) R_{h h}(i-j)=r_{y h}(j) \text { for } j=0,1, \ldots, N_{x} .
$$

So finall relationship is as follows:

$$
x(t)=-\sum_{i=0}^{N_{x}} x(i) h(t-i) .
$$

This is the case in a closed communication standard such as ZigBee [12], when one cannot analytically determine the delay generated as a result of the implementation of the software mechanisms. 
By the standard analysis, one can estimate the delays caused by the mechanisms contained in the MAC layer [13]. Taking under consideration properties of the CSMA/CA mechanism and because of the use of $\mathrm{O}$ -QPSK modulation connected with a direct spread spectrum (DSSS) transmission one can estimate the probability density function of the delay caused by these mechanisms. It is described by a normal distribution with parameters $(2,0.5)[2,3,14]$.

With a distribution of the density function of delay occurring in point to point configuration in the conditions of separation from any disturbance factors one can determine the density function of delay caused by software mechanisms as shown in Example 1.

Example 1. Histogram of total delay was achieved by performing measurements in ideal conditions obtained through the use of an insulating chamber. ZigBee wireless network using point to point configuration was used. The transmission took place over a distance of $15 \mathrm{~cm}$ and the data packet of a length of $1 \mathrm{~B}$ was sent. The resulting distribution has all the characteristics of a normal distribution with parameters $(10.5,0.5)$.

This phenomenon can be explained by the fact that the histogram describing the distribution of the total delay is obtained by convolution of distribution with the Dirac function shifted by a delay value $\tau_{\mathrm{S}}=8 \mathrm{~ms}$.

The convolution of an arbitrary function with the Dirac function is shown in the expression (29), and convolution of an arbitrary function with the Dirac function shifted by a distance $o$ is shown in the expression (30):

$$
\begin{aligned}
& \delta(t) \otimes f(t)=\int_{-\infty}^{+\infty} \delta(\tau) f(t-\tau) \mathrm{d} \tau=f(t), \\
& \delta(t+o) \otimes f(t)=\int_{-\infty}^{+\infty} \delta(\tau+o) f(t-\tau) \mathrm{d} \tau \\
& \quad=f(t+o) .
\end{aligned}
$$

Because thus obtained value corresponds to the delay resulting from the treatment of both encapsulation of data and decapsulation of data one can assume that the delay associated with the transmitter and the receiver is a half of this value.

In more complex cases, the structure of the wireless network, one must also take into account the delay associated with the presence of intermediary network devices.

\section{Conclusions}

Presented in the paper method of modelling communication delays occurring in wireless networks using random string of function is an extension of the concept presented in [6]. It is a new modelling approach allowing for probabilistic description of the tasks in network devices, regardless of the role that they play (transmitters, receivers, an intermediary network device) and the use of the delta function series to describe the message transmission time between the transmitter and the receiver. Probability density function was applied in the description of the delays. The conducted considerations were that the probability density function of the total delay can be described by a convolution of the probability density function of the delay introduced by the transmitter as a result of the task of data encapsulation and access to the media and the receiving device including the possible occurrence of intermediary devices.

As a result of transformations an expression called random string function was obtained. Random string function consists of the sum of the products of the following factors. The first is a density function of delay occurring while performing the task of encapsulation and tasks aimed at gaining access to the medium, shifting background of a certain value of delay, and the second one is weighting factor.

The model takes into account structural factors of communication devices. The presented model of the delays has been created for use in a wireless network using ZigBee standard. However due to the similarities in many wireless standards presented model may be used in any transmission network.

\section{References}

[1] P. Kielan, T. Trawiński, Electr. Rev. 86, 61 (2010) (in Polish).

[2] M. Hassan, R. Jain, High Performance TCP/IP Networking: Concepts, Issues, and Solutions, PrenticeHall, Upper Saddle River 2003.

[3] L. Larry, R. Peterson, S. Bruce, I. Dave, Computer Networks - A System Approach, 5th ed., Morgan Kaufmann, Burlington 2011.

[4] S. Andrew, D. Tanenbaum, J. Wetherall, Computer Networks, 5th ed., Prentice-Hall, New York 2011.

[5] J. Jakubiec, Errors and Data Uncertainties in Measurement-Control Systems, Vol. 76, SUT, Gliwice 2010 (in Polish).

[6] J. Jakubiec, B. Krupanek, M. Grygiel, Electrics, Vol. 216, SUT, Gliwice 2012 (in Polish).

[7] T. Topór-Kaminski, R. Żurkowski, M. Grygiel, Acta Phys. Pol. A 120, 748 (2011).

[8] D. Hardt, The Dirac Delta Function and Convolution, MIT, Cambridge 2004.

[9] E. Michta, Communications Models of MeasurementControl Network Systems, Vol. 38, Zielona Góra Technical University, Zielona Góra 2000 (in Polish).

[10] K. Gut, Bull. Pol. Acad. Sci., Techn. Sci. 59, 395 (2011).

[11] D. Heymann, M. Lichtblau, S. Rathmann, Elektronik Plus 6, 7 (1993).

[12] V. Candy James, Model-Based Signal Processing, Lawrence Livermore National Laboratory, University of California, Santa Barbara, CA 2006.

[13] ZigBee Specification v1.0, IEEE Standard for Information (LR-WPAN), New York 2003.

[14] A. Khan, K. Saad, A. Fahad, Performance Analysis of a ZigBee Beacon Enabled Cluster Tree Network, IEEE 978-1-4244-4361-1/09, 2009. 